\title{
Acidentes ocupacionais com exposição a material biológico: retrato de uma realidade*
}

\author{
Occupational injury involving exposure to \\ biological fluids: a portrait of reality
}

Maria Aparecida do C. Dias ${ }^{1}$, Alcyone A. Machado², Branca M. O. Santos ${ }^{3}$

\begin{abstract}
RESUMO
Objetivo: caracterizar os acidentes ocupacionais por exposição à material biológico notificados nos serviços de referência da micro região de Votuporanga- SP, no período de 2001 a 2005. Material e método: trata-se de uma pesquisa descritiva, do tipo levantamento documental retrospectivo, dos casos de acidentes ocupacionais por exposição a material biológico obtidos das 273 fichas de notificação do SINABIO encaminhadas aos bancos de dados do Serviço de Vigilância Epidemiológica da Secretaria Municipal de Saúde de Votuporanga-SP e da Direção Regional de Saúde XXII (DIRXXII) de São José do Rio Preto-SP. Resultados: dos 273 acidentes notificados, $78,4 \%$ ocorreram em trabalhadores do sexo feminino e $69,6 \%$ entre 20 e 40 anos. Votuporanga foi o município que mais notificou (89,0\%) e o auxiliar de enfermagem a categoria mais exposta $(48,4 \%)$. Em $99,6 \%$ dos casos, o atendimento se deu dentro das 72 horas. A exposição mais frequente foi a percutânea $(86,8 \%)$, o sangue o material biológico de maior contato $(98,5 \%)$ e a agulha com lúmen a que mais provocou acidentes $(72,1 \%)$. Dos acidentados, $87,5 \%$ eram vacinados contra HBV e desses, $67,0 \%$ não haviam realizado o anti-HBs. Em $16,1 \%$ das notificações a fonte HIV era desconhecida e em $78,8 \%$ era conhecida para o HBV. Para o HCV, detectouse $78,8 \%$ de sorologias de pacientes-fonte, das quais $3,3 \%$ eram positivas, sendo que $25,9 \%$ dos com fonte desconhecida não fizeram acompanhamento. Conclusões: os dados indicam a necessidade de elaboração de um plano de intervenção local, com a aquiescência da comunidade que é, inquestionavelmente, co-responsável pelas propostas de mudança.
\end{abstract}

Palavras-chave: Acidentes Ocupacionais. Risco por Agentes Biológicos. HIV. Hepatite. Notificação de Acidentes de Trabalho.

* Parte da dissertação de mestrado "Perfil epidemiológico dos acidentes ocupacionais por exposição a material biológico da micro-região de Votuporanga, período de 2001 a 2005. Universidade de Franca, 2007.

'Enfermeira. Mestre em Promoção de Saúde pela Universidade de Franca-UNIFRAN.

2Professora Associada da Faculdade de Medicina de Ribeirão Preto-USP. Departamento de Clínica Médica.

${ }^{3}$ Professora Associada em Enfermagem-USP. Docente do Mestrado em Promoção de Saúde da Universidade de FrancaUNIFRAN.
Correspondencia:

Branca Maria de Oliveira Santos Programa de Mestrado em Promoção de Saúde Universidade de Franca Av. Dr. Armando Salles de Oliveira, 201 14404-600 - Franca/SP Fone:( 0xx16)-36307877 e-mail: brancamosantos@gmail.com

Artigo recebido em 30/08/2011 Aprovado para publicação em 02/01/2012 


\section{Introdução}

Os acidentes que ocorrem pelo exercício do trabalho, a serviço da empresa, e que podem provocar lesões corporais, perturbação funcional e doenças que causem a morte e/ou perda ou redução permanente ou temporária da capacidade laboral para o trabalho, podem acontecer no local de trabalho, nos intervalos ou a caminho. ${ }^{1,2,3}$ Eles têm sido relacionados aos riscos oriundos da organização social do trabalho, desencadeando a participação dos trabalhadores nas questões de saúde e segurança no trabalho ${ }^{4}$ e têm representado um grave problema de saúde coletiva no Brasil.

Dentre os riscos ocupacionais destacam-se os biológicos, causados por microrganismos, culturas celulares e endoparasitas humanos, incluindo também microrganismos geneticamente modificados, que podem causar doenças infecciosas pelo contato com sangue ou fluidos corpóreos, na dependência de variáveis relacionadas ao agente, hospedeiro ou atividade ocupacional, sendo de vital importância a identificação, no ambiente de trabalho, dos fatores ou situações com potencial de dano, bem como a avaliação do risco para se estimar a possibilidade de ocorrência e a gravidade do dano. ${ }^{5,6}$

As exposições que podem colocar o profissional em risco de infecção por esses agentes são a injúria percutânea e o contato da membrana mucosa ou pele não íntegra com sangue, tecido ou outros fluidos corporais potencialmente infecciosos. O sêmen e secreções vaginais também são considerados potencialmente infecciosos. ${ }^{7}$

Embora tenha sido documentado que pelo menos 60 patógenos podem ser transmitidos através da exposição aos fluidos corpóreos, muita ênfase tem sido dada à epidemiologia e à prevenção das exposições ao Vírus da Imunodeficiência Humana (HIV), Vírus da hepatite B (HBV) e Vírus da hepatite C (HCV), que passaram a representar um sério problema de saúde pública. ${ }^{8}$

Os principais fatores de risco para transmissão ocupacional da infecção pelo HIV estão relacionados ao próprio acidente, à fonte contaminadora e ao acidentado. São também fatores determinantes, o tipo de exposição (percutânea, mucosa ou cutânea), o tipo de fluido envolvido (sangue ou fluidos corpóreos), a concentração de HIV no fluido, a gravidade da exposição (profundidade, extensão e tecido envolvido) e fatores físicos (temperatura, $\mathrm{pH}$, e umidade). A maioria dos casos de transmissão ocupacional ocorre após exposição a amostras frescas e embora o risco de transmissão seja baixo (0,3\%), medidas preventivas devem ser adotadas visando à minimização das consequências. ${ }^{9}$

$\mathrm{O}$ risco à infecção pelo HBV está primariamente relacionado ao grau de contato com sangue e ao estado antigênico (antígeno e do $\mathrm{HBV}-\mathrm{HBeAg}$ ) da pessoa-fonte. Estudos têm demonstrado um risco entre $22 \%$ a $31 \%$, com sangue reagente para $\mathrm{HBsAg}$ ( antígeno de superfície do HBV) e HBeAg. Na transmissão ocupacional pelo $\mathrm{HCV}$, a média de soroconversão do anticorpo contra hepatite C (anti-HCV), após a exposição percutânea acidental a uma pessoafonte $\mathrm{HCV}$ positiva, é de 1,8\%. ${ }^{7}$

Mesmo diante da escassez de dados sistematizados sobre os acidentes ocupacionais com exposição a esses patógenos, dados têm apontado que desde o início da epidemia de aids, até 2002 , foram publicados em todo o mundo 106 casos comprovados e 238 casos prováveis de trabalhadores da área da saúde contaminados pelo HIV por acidente de trabalho. ${ }^{10,11}$

No Brasil, dados fornecidos por órgãos de saúde, indicam quatro casos de contaminação pelo HIV após exposição ocupacional ${ }^{12}$ e outros seis de notificação de infecção que se encontram em investigação. ${ }^{13}$ A prevalência de soroconversão de hepatite B apresenta taxa de 7,9\%, menor apenas que a República Dominicana $(21,4 \%)$, com maiores taxas na Amazônia. ${ }^{14}$ A prevalência de anti-HCV em trabalhadores da área da saúde varia de $0 \%$ a $1,7 \%$, sendo maior $(1,4 \%)$ em trabalhadores de centros de hemodiálise. ${ }^{15}$

Analisados de forma isolada, esses dados, no entanto, não refletem a realidade da contaminação de trabalhadores pelos referidos patógenos, reforçando a ideia de que além de as notificações de casos de infecção por exposição ocupacional serem passivas, a documentação dos casos pode ser difícil quando não relatadas logo após a ocorrência.

Em 1999, no Estado de São Paulo, foi implantado o Sistema de Notificação de Acidentes Biológicos (SINABIO) pela Secretaria Municipal de Saúde de São Paulo, que passou a contemplar os acidentes com profissionais de saúde, bombeiros, policiais, profissionais de limpeza de serviços de saúde, cuidadores domiciliares e indivíduos em situação de atendimento de saúde eventual, bem como as ações a serem seguidas. ${ }^{16,17}$

A notificação deve ser feita no momento em que o acidente ocorre, atentando para a avaliação do acidente, situação sorológica do paciente fonte, coleta 
de material para determinação da necessidade de quimioprofilaxia e seguimento clínico-laboratorial do acidentado, dentre outras.

Frente ao exposto, o objetivo do estudo foi caracterizar os acidentes ocupacionais com exposição à material biológico notificados nos serviços de referência da micro região de Votuporanga- SP, no período de 2001 a 2005, quanto à ocupação dos trabalhadores acidentados, local de trabalho, agente causador, tipo e local de exposição, material biológico de contato, tempo decorrido entre o acidente e a notificação, avaliação vacinal do acidentado e estado sorológico dos envolvidos.

\section{Material e Método}

Trata-se de uma pesquisa descritiva, do tipo levantamento documental retrospectivo, dos casos de acidentes ocupacionais com exposição a material biológico, notificados e obtidos das 273 fichas de notificação do SINABIO, encaminhadas aos bancos de dados do Serviço de Vigilância Epidemiológica da Secretaria Municipal de Saúde do município de Votuporanga e da Direção Regional de Saúde XXII (DIRXXII) de São José do Rio Preto-SP, que é referência à micro-região de Votuporanga.

Com a municipalização da saúde, por volta do ano 2000, os acidentes ocupacionais ocorridos em Votuporanga e nos 15 municípios circunvizinhos passaram a ser encaminhados e atendidos em um Ambulatório de Especialidades (AE), que servia de referência para todas as unidades de saúde dos municípios, seguindo um atendimento baseado no protocolo do Ministério da Saúde, Secretaria de Vigilância em Saúde e Programa Nacional de DST/aids, denominado "Recomendações para atendimento e acompanhamento de exposição ocupacional a material biológico: HIV e hepatites B e C". ${ }^{18}$

Os dados foram coletados através de um instrumento contendo os dados de identificação do trabalhador acidentado (nome, idade, sexo, endereço, local do trabalho, categoria profissional, data e horário do acidente e data e horário do atendimento) e do acidente (local de exposição, tipo de material biológico, agente causador, circunstância do acidente, estado sorológico e vacinal do acidentado e estado sorológico da fonte) e foram registrados e elaborados através do programa Microsoft Excel e submetidos à análise descritiva.

O projeto foi aprovado pelo Comitê de Ética em Pesquisa da Universidade de Franca, em 28/04/06, sob o n. ${ }^{\circ}$ 033/06, de acordo com a Resolução no 196/96 do Conselho Nacional de Saúde.

\section{Resultados}

Dos 273 acidentes ocupacionais com exposição à material biológico notificados no período estudado, a maior frequência ocorreu no ano de 2002 (23,8\%), seguida pelos anos de 2005 (22,7\%), 2003 $(19,4 \%), 2004(18,7 \%)$ e $2001(15,4 \%)$. A predominância de notificações ocorreu no município de Votuporanga $(89,0 \%)$, com destaque para o número de municípios que se mantiveram silenciosos durante o período estudado (Tabela 1).

Do total de trabalhadores acidentados, 214 $(78,4 \%)$ eram do sexo feminino, cuja maioria foi representada por profissionais de enfermagem. Podese observar um aumento de notificações de acidentes em profissionais do sexo masculino em 2005, representados, em sua maioria, por $5(25,0 \%)$ médicos e 4 dentistas $(20,0 \%)$.

Com relação à idade, a maior frequência ficou entre 20 e 40 anos $(69,6 \%)$ e de 40 a 50 anos $(22,0 \%)$. Dos cinco casos $(1,8 \%)$ em profissionais com idades inferiores a 20 anos, um era estudante, um auxiliar de odontologia, um técnico de enfermagem e dois do serviço de limpeza. Os casos ocorridos em indivíduos com idades acima de 50 anos, foram representados por quatro médicos e um dentista, todos do sexo masculino (Tabela 2).

Em relação ao local de trabalho do acidentado, a Tabela 3 mostra que $179(65,6 \%)$ acidentes ocorreram em hospitais de Votuporanga, dos quais 132 $(73,7 \%)$ num hospital filantrópico de grande porte e $47(26,3 \%)$ numa entidade privada. Os acidentes ocorridos em Unidades Básicas de Saúde representaram $29,3 \%$, englobando toda micro-região. Não foi encontrada nenhuma notificação em hospitais da região e, na maioria das fichas, também não havia informação do setor onde o mesmo ocorreu, ainda que houvesse um espaço específico para este dado.

Dentre as notificações relacionadas ao tipo de exposição, pode-se observar, pela Tabela 4, uma elevada frequência de acidentes percutâneos, 237 (86,8\%), merecendo destaque as categorias de auxiliar de enfermagem com 132 (48,4\%) e médico com 35 (12,8\%).

Em relação ao tipo de material biológico com o qual o acidentado entrou em contato, o sangue foi o fluido corporal mais notificado $(98,5 \%)$. O dispositivo que mais provocou acidente foi a agulha com lúmen, 
Tabela 1

Acidentes com exposição à material biológico notificados no Serviço de Vigilância Epidemiológica da microregião de Votuporanga/SP, segundo o município e período de ocorrência. Votuporanga, 2001 a 2005.

\begin{tabular}{|c|c|c|c|c|c|c|c|}
\hline \multirow[b]{2}{*}{ Município } & \multicolumn{5}{|c|}{ Período de ocorrência } & \multicolumn{2}{|c|}{ Total } \\
\hline & 2001 & 2002 & 2003 & 2004 & 2005 & $\mathbf{N}^{\circ}$ & $\%$ \\
\hline Álvares Florence & 0 & 1 & 0 & 0 & 0 & 1 & 0,4 \\
\hline Américo de Campos & 0 & 0 & 1 & 0 & 1 & 2 & 0,7 \\
\hline Cardoso & 0 & 1 & 1 & 6 & 7 & 15 & 5,5 \\
\hline Cosmorama & 0 & 1 & 0 & 1 & 2 & 4 & $\mathbf{1 , 5}$ \\
\hline Floreal* & 0 & 0 & 0 & 0 & 0 & $\mathbf{0}$ & $\mathbf{0 , 0}$ \\
\hline General Salgado & 0 & 0 & 2 & 0 & 0 & 2 & 0,7 \\
\hline Macaubal & 0 & 0 & 1 & 0 & 0 & 1 & 0,4 \\
\hline Magda* & 0 & 0 & 0 & 0 & 0 & $\mathbf{0}$ & $\mathbf{0 , 0}$ \\
\hline Monções* & 0 & 0 & 0 & 0 & 0 & $\mathbf{0}$ & $\mathbf{0 , 0}$ \\
\hline Nhandeara* & 0 & 0 & 0 & 0 & 0 & $\mathbf{0}$ & $\mathbf{0 , 0}$ \\
\hline Parisi & 1 & 1 & 0 & 0 & 0 & 2 & 0,7 \\
\hline Pontes Gestal & 0 & 0 & 1 & 0 & 0 & 1 & 0,4 \\
\hline Riolândia & 0 & 1 & 1 & 0 & 0 & 2 & 0,7 \\
\hline Sebastianópolis do Sul* & 0 & 0 & 0 & 0 & 0 & $\mathbf{0}$ & $\mathbf{0 , 0}$ \\
\hline Valentim Gentil* & 0 & 0 & 0 & 0 & 0 & $\mathbf{0}$ & $\mathbf{0 , 0}$ \\
\hline Votuporanga & 41 & 60 & 46 & 44 & 52 & 243 & 89,0 \\
\hline Total & $\begin{array}{c}42 \\
(15,4 \%)\end{array}$ & $\begin{array}{c}65 \\
(23,8 \%)\end{array}$ & $\begin{array}{c}53 \\
(19,4 \%)\end{array}$ & $\begin{array}{c}51 \\
(18,7 \%)\end{array}$ & $\begin{array}{c}62 \\
(22,7 \%)\end{array}$ & 273 & 100,0 \\
\hline
\end{tabular}

*Municípios silenciosos

Tabela 2

Acidentes com exposição à material biológico notificados no Serviço de Vigilância Epidemiológica da microregião de Votuporanga/SP, segundo a idade e o período de ocorrência. Votuporanga, 2001 a 2005.

\begin{tabular}{|c|c|c|c|c|c|c|c|c|}
\hline \multirow[b]{2}{*}{ Idade } & & \multicolumn{5}{|c|}{ Período de ocorrência } & \multicolumn{2}{|c|}{ Total } \\
\hline & & 2001 & 2002 & 2003 & 2004 & 2005 & $\mathbf{N}^{\circ}$ & $\%$ \\
\hline$<$ & 20 & 0 & 1 & 1 & 2 & 1 & 5 & 1,8 \\
\hline 20 & 30 & 11 & 21 & 17 & 22 & 22 & 93 & 34,1 \\
\hline 30 & 40 & 19 & 24 & 16 & 16 & 22 & 97 & 35,5 \\
\hline 40 & 50 & 10 & 14 & 14 & 8 & 14 & 60 & 22,0 \\
\hline 50 & 60 & 2 & 4 & 4 & 1 & 2 & 13 & 4,8 \\
\hline $60 \mid$ & + & 0 & 1 & 1 & 2 & 1 & 5 & 1,8 \\
\hline Total & & 42 & 65 & 53 & 51 & 62 & 273 & 100,0 \\
\hline
\end{tabular}




\section{Tabela 3}

Acidentes com exposição a material biológico notificados no Serviço de Vigilância Epidemiológica da Microregião de Votuporanga/SP, segundo o local de trabalho e o período de ocorrência. Votuporanga, 2001 a 2005.

\begin{tabular}{|c|c|c|c|c|c|c|c|}
\hline \multirow[b]{2}{*}{ Local de trabalho } & \multicolumn{5}{|c|}{ Período de ocorrência } & \multicolumn{2}{|c|}{ Total } \\
\hline & 2001 & 2002 & 2003 & 2004 & 2005 & $\mathbf{N}^{\mathbf{o}}$ & $\%$ \\
\hline Hospital* & 27 & 48 & 34 & 33 & 37 & 179 & 65,6 \\
\hline UBS* & 13 & 16 & 16 & 17 & 18 & 80 & 29,3 \\
\hline $\mathrm{AE}^{*}$ & 0 & 0 & 2 & 0 & 2 & 4 & 1,4 \\
\hline Domicílio & 1 & 0 & 0 & 0 & 0 & 1 & 0,4 \\
\hline LSVP* & 0 & 0 & 0 & 0 & 1 & 1 & 0,4 \\
\hline Consultório & 1 & 0 & 0 & 1 & 2 & 4 & 1,4 \\
\hline Viatura & 0 & 0 & 0 & 0 & 1 & 1 & 0,4 \\
\hline Presídio & 0 & 0 & 1 & 0 & 1 & 2 & 0,7 \\
\hline $\mathrm{CBL}^{*}$ & 0 & 1 & 0 & 0 & 0 & 1 & 0,4 \\
\hline Total & 42 & 65 & 53 & 51 & 62 & 273 & 100,0 \\
\hline
\end{tabular}

*(Hospital) Santa Casa de Misericórdia e Casa de Saúde e Maternidade Nossa Senhora Aparecida, ambas de Votuporanga, (UBS) Unidade Básica de Saúde, (AE) Ambulatório de Especialidades, (LSVP) Lar São Vicente de Paulo e (CBL) Companhia Brasileira de Lixo

\section{Tabela 4}

Acidentes com exposição a material biológico notificados no Serviço de Vigilância Epidemiológica da microregião de Votuporanga/SP, segundo a categoria profissional e o tipo de exposição. Votuporanga, 2001 a 2005.

\begin{tabular}{|c|c|c|c|c|c|c|}
\hline \multirow[b]{2}{*}{ Categoria profissional } & \multicolumn{4}{|c|}{ Tipo de exposição } & \multicolumn{2}{|c|}{ Total } \\
\hline & Percutânea & Mucosa Ocular & Pele Íntegra & Pele Ñ Íntegra & $\mathbf{N}^{\mathbf{o}}$ & $\%$ \\
\hline Auxiliar de Enfermagem & 119 & 10 & 2 & 1 & 132 & 48,4 \\
\hline Médico & 31 & 2 & 2 & 0 & 35 & 12,8 \\
\hline Pessoal da Limpeza & 21 & 1 & 0 & 0 & 22 & 8,1 \\
\hline Estudante & 14 & 0 & 1 & 1 & 16 & 5,9 \\
\hline Dentista & 12 & 1 & 1 & 0 & 14 & 5,1 \\
\hline Técnico de Enfermagem & 12 & 1 & 0 & 1 & 14 & 5,1 \\
\hline Auxiliar de Odontologia & 10 & 0 & 1 & 0 & 11 & 4,0 \\
\hline Enfermeiro & 5 & 3 & 0 & 1 & 9 & 3,3 \\
\hline Policial & 1 & 0 & 0 & 3 & 4 & 1,4 \\
\hline Secretária & 2 & 0 & 0 & 1 & 3 & 1,1 \\
\hline Funcionário da Lavanderia & 2 & 0 & 0 & 0 & 2 & 0,7 \\
\hline Funcionário da Manutenção & 2 & 0 & 0 & 0 & 2 & 0,7 \\
\hline Recepcionista & 0 & 1 & 0 & 1 & 2 & 0,7 \\
\hline Auxiliar de Laboratório & 2 & 0 & 0 & 0 & 2 & 0,7 \\
\hline Lixeiro & 1 & 0 & 0 & 0 & 1 & 0,4 \\
\hline Fisioterapeuta & 1 & 0 & 0 & 0 & 1 & 0,4 \\
\hline Bombeiro & 0 & 0 & 0 & 1 & 1 & 0,4 \\
\hline Agente de Saúde & 1 & 0 & 0 & 0 & 1 & 0,4 \\
\hline Atendente de Enfermagem & 1 & 0 & 0 & 0 & 1 & 0,4 \\
\hline Total & $\begin{array}{c}237 \\
(86,8 \%)\end{array}$ & $\begin{array}{c}19 \\
(7,0 \%)\end{array}$ & $\begin{array}{c}7 \\
(2,5 \%)\end{array}$ & $\begin{array}{c}10 \\
(3,7 \%)\end{array}$ & 273 & 100,0 \\
\hline
\end{tabular}


com $197(72,1 \%)$. Ao se relacionar com a circunstância do acidente, 129 (65,5\%) ocorreram durante a administração de medicamentos, $31(15,7 \%)$ por descarte inadequado de material, $28(14,2 \%)$ durante procedimento cirúrgico, $2(1 \%)$ por reencape de agulhas e $7(3,6 \%)$ por outros motivos.

Pelas datas e horários do acidente e do atendimento, foi possível observar que $99,6 \%$ dos acidentados foram atendidos dentro das primeiras 72 horas após o acidente, e a Tabela 5 demonstra que 239 $(87,5 \%)$ dos acidentados estavam vacinados contra a Hepatite B, ainda que $160(67,0 \%)$ não possuíam registro da realização do teste sorológico anti-HBs para confirmação da resposta vacinal. Não foi encontrada também nenhuma referência nas fichas de notificação acerca do uso de imunoglobulina hiperimune contra hepatite B (IGHAHB). Em um dos casos detectados, mesmo após o esquema vacinal completo (três doses), o anti-HBs foi negativo.

O estado sorológico positivo do acidentado no momento do acidente foi notificado em seis fichas, sendo uma para HIV (médico), outra para o HBV (dentista) e quatro para o HCV (dentista, auxiliar de enfermagem, escriturário e pessoal da limpeza). Foi possível detectar também que em 15 (5,5\%), 22 (8,2\%) e $20(7,3 \%)$ não havia nenhuma anotação dos estados sorológicos para HIV, HBV e HCV, respectivamente, significando fichas incompletas.

A avaliação do estado sorológico do pacientefonte possibilitou a informação de que em $229(83,9 \%)$ dos casos notificados havia referência de sorologia antiHIV, dos quais $218(95,2 \%)$ possuíam marcador sorológico negativo, e 11 (4,8\%) positivo. Quanto à hepatite B, foi possível detectar 210 (97,7\%) dos casos com resultado negativo e 2,3\% com positivo. Já em relação ao HCV, 208 (96,7\%) foram negativos e 3,3\% positivos. O número de notificações caracterizando fonte desconhecida foi de $44(16,1 \%)$ para o HIV e 58 $(21,2 \%)$ para o HBV e HCV, respectivamente.

Das $11(4,8 \%)$ notificações com positividade sorológica no paciente-fonte, $5(45,6 \%)$ foram identificadas em auxiliares de enfermagem, $2(18,3 \%) \mathrm{em}$ auxiliares de odontologia e uma $(9,1 \%)$ em técnico de enfermagem, profissional da limpeza, aluno e médico, respectivamente.

Dos 44 casos de fonte desconhecida para HIV, $19(43,2 \%)$ foram em profissionais da limpeza, nove $(20,5 \%)$ em auxiliares de enfermagem, cinco $(11,4)$ $\%$ em auxiliares de odontologia, três $(6,8 \%)$ em dentistas, dois $(4,5 \%)$ em pessoal da manutenção e seis $(13,6 \%)$ em enfermeiro, aluno, lixeiro, técnico de enfermagem, agente comunitário de saúde e médico.

Das cinco notificações de profissionais que tiveram contato com fonte positiva para HBV, $4(80,0 \%)$ eram auxiliares de enfermagem e a outra era uma recepcionista. Um dos auxiliares de enfermagem era vacinado, mas não havia realizado anti-HBs e não fez acompanhamento com sorologias para esclarecer se houve ou não contaminação pelo referido vírus. Os demais fizeram acompanhamento e não houve soroconversão.

Pelas fichas com notificação de fonte desconhecida para HBV, observou-se que a categoria profissional mais atingida foi a de auxiliar de enfermagem, com $20(34,5 \%)$. A categoria profissional da limpeza ficou com 19 (32,8\%) notificações, a do auxiliar de odontologia com seis $(10,3 \%)$ e $13(22,4 \%)$ foram representadas por alunos, dentistas, funcionários do serviço de manutenção, técnico de enfermagem, enfermeiro, médico, agente comunitário de saúde e lixeiro.

\section{Tabela 5}

Situação vacinal contra a hepatite B dos acidentados por exposição a material biológico notificados no Serviço de Vigilância Epidemiológica da micro-região de Votuporanga/SP, segundo o período de ocorrência. Votuporanga, 2001 a 2005.

\begin{tabular}{|c|c|c|c|c|c|c|c|}
\hline \multirow[b]{2}{*}{ Vacinação Hepatite b } & \multicolumn{5}{|c|}{ Período de ocorrência } & \multicolumn{2}{|c|}{ Total } \\
\hline & 2001 & 2002 & 2003 & 2004 & 2005 & $\mathbf{N}^{\mathbf{o}}$ & $\%$ \\
\hline Vacinado & 35 & 53 & 45 & 48 & 58 & 239 & 87,5 \\
\hline Não Vacinado & 7 & 12 & 8 & 3 & 4 & 34 & 12,5 \\
\hline Total & 42 & 65 & 53 & 51 & 62 & 273 & 100,0 \\
\hline
\end{tabular}


Do total dos 58 casos notificados de fonte desconhecida para o HBV, 44 (75,9\%) dos trabalhadores eram vacinadas com as três doses da vacina. $\mathrm{O}$ mais preocupante é que dos vacinados, somente $15(34,1 \%)$ tinham comprovante de imunização contra o HBV. Os $14(24,1 \%)$ trabalhadores não vacinados foram acompanhados, sem que houvesse registro de confirmação de infecção.

Dos $29(65,9 \%)$ profissionais vacinados e que não realizaram o anti-HBs, 14 (48,3\%) não fizeram acompanhamento para confirmar se houve ou não a infecção pelo HBV e 15 (51,7\%) realizaram seguimento sem, no entanto, ter sido encontrada notificação de infecção.

Das sete notificações positivas para o $\mathrm{HCV}$ $(3,3 \%)$, as categorias profissionais mais atingidas foram o auxiliar de enfermagem $(28,6 \%)$, técnicos de enfermagem (34,5\%), pessoal de limpeza $(32,8 \%)$ e auxiliar de odontologia (10,3\%). Pelos dados observase uma situação semelhante em relação à hepatite $\mathrm{B}$.

\section{Discussão}

A ocorrência de menor número de acidentes ocupacionais notificados em 2001 pode ser justificada pelo fato de ter sido o ano de implantação do SINABIO na referida região, ou ainda pelo despreparo das equipes responsáveis pelo atendimento dos acidentes, levando, inclusive, a uma possível subnotificação. O maior número de notificações no ano de 2002, com diminuição a partir de 2003 e nova elevação em 2005, demonstra uma realidade um pouco diferente da redução do número de notificações em 2002, divulgada pelo Boletim Epidemiológico, dos acidentes com material biológico ocorridos no estado de São Paulo. ${ }^{16}$ Essa redução pode ser justificada por uma possível queda das notificações, pelo grande número de notificações inconsistentes e elevado percentual de casos sem conclusão, e não a uma real redução dos acidentes.

A predominância de notificações no município de Votuporanga $(89,0 \%)$ pode ter ocorrido pelo fato de o município ser referência para os demais e por ser o mais populoso.

Pesquisadores têm revelado altos índices de subnotificação de acidentes entre trabalhadores de saúde, destacando como principais causas, as crenças dos trabalhadores, a falta de informações sobre o risco de contaminação e a forma de realização da notificação, alertando para a importância do registro para o planejamento de estratégias preventivas, cria- ção de programas de educação em serviço e necessidade de treinamento e supervisão quanto ao registro dos mesmos. ${ }^{19-24}$

A maior ocorrência de acidentes ocupacionais em trabalhadores do sexo feminino, representados em sua maioria por profissionais de enfermagem, corrobora com os resultados de com outros estudos ${ }^{21,25,26}$, e pode ser devido ao fato de que cerca de $90 \%$ do seu contingente no Brasil ser formado por mulheres. ${ }^{27}$ Já o aumento do número de acidentes em 2005 entre profissionais do sexo masculino, representados principalmente por profissionais da medicina e odontologia, pode ser atribuído pela hegemonia de profissionais desse sexo nessas profissões e pelo tipo de atividade profissional que exercem, levando a uma maior exposição aos riscos de acidentes perfurocortantes.

A maior frequência de acidentes em profissionais com idades entre 20 a 50 anos também foi verificada no estudo de Caixeta e Barbosa-Branco. ${ }^{25}$

$\mathrm{O}$ fato de a maioria dos acidentes notificados $(65,6 \%)$ ter ocorrido em instituições hospitalares de Votuporanga, sem nenhum registro em outro hospital da micro-região, indica uma possível falta de identificação do local do acidente, uma vez que, na maioria das vezes, a anotação referia apenas como tendo ocorrido em Unidade de Saúde, não especificando se hospitalar ou na Rede Básica.

$O$ fato de a maioria das fichas de notificação não conter referência do setor onde ocorreu o acidente, ainda que existisse um espaço específico para preenchimento, pode representar um importante indicador de risco e direcionar a condução de medidas preventivas, educativas e até organizacionais. O pequeno número de acidentes ocorridos em consultórios particulares levantou o questionamento sobre a possibilidade de subnotificação, pois se sabe que existem consultórios médicos e odontológicos particulares onde são realizados muitos procedimentos invasivos.

A supremacia dos auxiliares de enfermagem como categoria profissional mais exposta a esse tipo de acidente corrobora com os resultados de outros estudos. ${ }^{28-31}$ Esses profissionais estão presentes em todos os momentos da assistência ao paciente, sendo a eles atribuídas as tarefas de cuidados diretos, administração de medicamentos, participação efetiva nas urgências e emergência e manuseio e preparo de instrumentos cirúrgicos após a utilização. ${ }^{27}$

O segundo lugar em notificações para a categoria de médico $(12,8 \%)$, talvez possa ser explicado pelo fato de ser a classe profissional mais próxima 
das unidades de saúde. O dentista, que ficou em quinto lugar de frequência, também está exposto a acidentes por materiais perfurocortantes durante o desenvolvimento de suas atividades profissionais. Bellissimo-Rodrigues, Bellissimo-Rodrigues e Macha$\mathrm{do}^{32}$ encontraram uma elevada frequência de acidentes durante o reencape de agulhas entre dentistas, alertando para a necessidade de um trabalho urgente de conscientização dos mesmos. No estudo de Caixeta e Barbosa-Branco ${ }^{25}$ os profissionais mais expostos aos acidentes por material biológico foram os médicos, dentistas e técnicos de laboratório.

A frequência de acidentes em profissionais de limpeza, terceiro lugar em notificações $(8,1 \%)$, foi discutida em estudos que chamaram a atenção para o agravante fato acerca do desconhecimento dos trabalhadores de saúde em relação à necessidade de descarte adequado de material perfurocortante, independentemente de estar ou não contaminado, e para o baixo conhecimento e aplicação de medidas preventivas na rotina diária pelos funcionários do serviço de higiene e limpeza hospitalar. ${ }^{28,33,34}$

A significativa frequência de notificação de acidentes entre estudantes $(5,9 \%)$ reforça a necessidade de um maior enfoque no ensino das normas de biossegurança, uma vez que nas unidades de saúde circula um número elevado de alunos de cursos de diferentes áreas do conhecimento. A gravidade desta situação foi confirmada em estudo com alunos de odontologia35, que constatou um percentual elevado de acidentes nos últimos períodos do curso, além de falhas nas medidas de precaução e de procura por serviço médico especializado.

Ainda que o auxiliar de odontologia tenha representado uma porcentagem de 4,0\% das notificações, chama a atenção que um profissional sofreu acidente percutâneo por duas vezes, com fonte sabidamente soropositiva para HIV, reforçando a gravidade do despreparo dessa categoria quanto às medidas de biossegurança.

A baixa notificação de acidentes em auxiliares de laboratório e bombeiros $(0,7 \%$ e $0,4 \%$, respectivamente), pode significar subnotificação ou levar à conjetura de que estão atentos às medidas de biossegurança, considerando o treinamento que recebem durante a sua formação.

As notificações de acidentes em policiais $(1,4 \%)$ foram relevantes e podem estar relacionadas ao tipo de atendimento que esses profissionais oferecem à população. Na única notificação da categoria profis- sional de fisioterapia, o acidente perfurocortante se deu com instrumental de aspiração, no momento em que o profissional realizava o procedimento. As categorias de manutenção, lixeiro, agente comunitário de saúde e atendente de enfermagem foram pouco notificadas, talvez por não realizarem procedimentos de risco junto a pacientes, apesar de poderem ser vítimas quando do descarte inadequado dos materiais perfurocortantes.

Nos três acidentes $(1,1 \%)$ ocorridos com profissionais da categoria de secretário de consultório, que normalmente responsabilizam-se pelas atividades de limpeza, desinfecção e esterilização de instrumentais clínicos, mesmo sem possuir formação para tal, os mesmos não estavam usando EPI e somente um era vacinado contra hepatite $\mathrm{B}$. Quanto às duas notificações para recepcionistas, um dos casos teve contato com fonte HBsAg positiva, não era vacinado contra a hepatite B e não estava usando EPI. Pelo acompanhamento sorológico desse profissional, não houve soroconversão.

Pelas informações relacionadas às datas e horários do acidente e do atendimento ao acidentado, pode-se detectar que uma auxiliar de enfermagem que sofreu acidente com material perfurocortante utilizado em fonte soropositiva para HIV, somente comunicou o acidente após 10 dias da ocorrência. Considerando o tempo decorrido entre os dois momentos, não foi indicado o uso de antirretrovirais. Este caso fez acompanhamento sorológico por seis meses e não houve soroconversão para HIV.

A exposição ocupacional deve ser tratada como emergência médica, preferencialmente nas primeiras horas após o acidente e no máximo até 72 horas. No caso de exposição ocupacional ao HIV, o uso do teste rápido no paciente-fonte justifica-se pelo fato de se ter um curto período de tempo para iniciar a terapêutica profilática com antirretrovirais, a fim de reduzir o risco de infecção em pelo menos $80 \%{ }^{36}$

O dispositivo que mais provocou acidente foi a agulha com lúmen $(72,1 \%)$. Os provocados por agulhas com lúmen/grosso calibre, lesão profunda, sangue visível no dispositivo ou agulha usada recentemente em artéria ou veia, representam a mais grave situação, principalmente quando o paciente-fonte é sabidamente soropositivo para HIV. ${ }^{30} \mathrm{O}$ fato de a agulha ter sido o artefato mais citado pelas enfermeiras $(97,6 \%)$ e pelas auxiliares de enfermagem $(97,1 \%)$ que se acidentam ${ }^{37}$, corrobora com os resultados de outros autores. ${ }^{26,38,39}$ 
Pela avaliação do estado vacinal do acidentado para o HBV detectou-se que a maioria era vacinada. A diminuição dos casos de não vacinados a partir de 2002, pode ter ocorrido em função da implementação de campanhas de vacinação junto aos trabalhadores do município, inclusive contra a hepatite $\mathrm{B}$, que desde setembro de 1998 faz parte do esquema rotineiro de vacinações para as crianças e profissionais de risco. ${ }^{40}$ Nesse sentido, no entanto, Gerberding ${ }^{41}$ chama a atenção para a importância de se realizar o teste para antiHBs de um a seis meses após a vacinação, principalmente em trabalhadores de saúde.

Em relação às notificações de fonte positiva para o HCV, observou-se uma situação semelhante em relação à hepatite $\mathrm{B}$. Esse marcador foi fonte desconhecida para $21,2 \%$ dos profissionais. Nesta situação recomenda-se acompanhar o profissional acidentado com a coleta dos marcadores sorológicos por seis meses, já que não existe medida preventiva pós- exposição desses eventos.

\section{Considerações Finais}

Pelos resultados encontrados foi possível observar a importância e a contribuição dos dados obti- dos das fichas de notificação avaliadas, sem as quais não seria possível delinear o retrato da realidade local em relação à situação dos acidentes ocupacionais com material biológico, mesmo considerando a impossibilidade de generalizações e o frequente número de subnotificações em alguns itens importantes.

Apesar de todas as possibilidades de informação oferecidas por um sistema implantado como ferramenta de trabalho, quase sempre ele apresenta uma baixa utilização das mesmas. O levantamento proposto possibilitou, assim, a visualização de aspectos até então sem avaliação e análise, tornando pública a situação dos acidentes com risco biológico no município. Possibilitou ainda a detecção de casos de trabalhadores que tiveram reincidência de acidentes, apontando os principais focos de atuação que poderão servir de subsídios para a elaboração de um plano de intervenção local. Nesse sentido, vale ressaltar a preocupação das autoras em devolver os resultados à Secretaria de Saúde Municipal com vistas à tomada de condutas pertinentes.

Ainda que os resultados representem apenas uma parcela da realidade, eles possibilitaram uma abordagem inicial acerca da problemática local e reforçam a necessidade de seguimento da avaliação realizada.

\begin{abstract}
Objective: to characterize occupational accidents with exposure to biological material reported in the referral services in the micro region of Votuporanga, SP, Brazil between 2001 and 2005. Material and Method: this is a descriptive survey, retrospective documentary survey of occupational accidents with exposure to biological material obtained from 273 report forms from the accident report system forwarded to the database of the Serviço de Vigilância Epidemiológica da Secretaria Municipal de Saúde de Votuporanga, SP and to the Direção Regional de Saúde XXII (DIRXXII) de São José do Rio Preto, SP, Brazil. Results: of the 273 reported accidents, $78.4 \%$ occurred with female workers and $69.6 \%$ were individuals between 20 and 40 years of age. Votuporanga was the city with the most reported accidents $(89.0 \%)$ and nursing auxiliaries were the most exposed professionals $(48.4 \%)$. In $99.6 \%$ of cases, care was provided within 72 hours. Percutaneous injury was the most frequent $(86.8 \%)$, blood was the biological material with which there was the most contact (98.5\%), and lumen needles accounted for $72.1 \%$ of the accidents. A total of $87.5 \%$ of the victims were vaccinated against Hepatitis B and $67.0 \%$ had not been tested for anti-HBs. The serological status against HIV of the source patient was unknown in $16.1 \%$ of the reports and in $78,8 \%$ was known for HBV. Hepatitis C serological tests for $78.8 \%$ of patient-source were verified, of which $3.3 \%$ tested positive, $25.9 \%$ whose source was unknown did not follow-up. Conclusions: data indicate the need to develop a local intervention plan with the consent of the community, which is undoubtedly co-responsible for proposals of change.
\end{abstract}

Keywords: Accidents, Occupational. Exposure to Biological Agents. HIV. Hepatitis. Occupational Accidents Registry. 


\section{Bibliografia}

1. Ribeiro MCSA. Acidentes de trabalho referidos por trabalhadores moradores na Região Metropolitana de São Paulo, 1994: um levantamento de base populacional. [Dissertação de Mestrado], Faculdade de Medicina, Universidade de São Paulo, São Paulo; 2000.

2. Brasil. Decreto n. 3048, de 06 de maio de 1999. Dispõe no artigo 131 define o acidente de trabalho. Diário Oficial da União, Brasília, seção II, 12 maio 1999.

3. Monteiro AL. Acidente do trabalho e doença ocupacional: conceito, processo deconhecimento e de execução e suas questões polêmicas. São Paulo: Saraiva; 1998.

4. Waismann W, Castro JAP. A evolução das abordagens da saúde e trabalho no capitalismo industrial. In: Teixeira $P$, Valle S. Biossegurança: uma abordagem multidisciplinar. Rio de Janeiro: Fiocruz; 1996. p. 15-25.

5. Silva FHAL, Rover G. Níveis de contenção física e classificação dos microrganismos por classe de riscos. In: Mastroeni MF. Biossegurança aplicada a laboratórios e serviços de saúde. São Paulo: Atheneu; 2004. p. 67-88.

6. Souza, M. Controle de riscos nos serviços de saúde. Acta Paul. Enferm. São Paulo 2000; 13: 197-202.

7. Centers for disease control and prevention. Updated U. S. Public health service guidelines for the management of occupational exposures to HBV, HCV and HIV and Recommendations for Postexposure Profhylaxis. Morbidity and mortality weekly report, United States 2001; 50: 1-8.

8. Gomes AC, Agy LL, Malaguti SE et al. Acidentes ocupacionais com material biológico e equipe de enfermagem de um hospital escola. Rev Enferm - UERJ, Rio de Janeiro 2009; 17: 220-3.

9. Oda LM, Rocha SS, Teixeira P. Aids como doença ocupacional. In: Teixeira P, Valle S. Biossegurança: uma abordagem multidisciplinar. Rio de Janeiro: Fiocruz; 1996. p. 249-53.

10. Health Protection Agency Center for Infections \& Collaborators. Occupational Transmission of HIV - Summary of Published Reports - Data to December 2002. London; march. 2005: 1-39.

11. Do AN, Ciesielski CA, Metler RP, Hammerr TA, Li J, Fleming PL. Occupational acquired human immunodeficiency vírus (HIV) infection: national case surveillance data during 20 years of the HIV epidemic in the United States. Infect Control Hosp Epidemiol 2003; 24: 86-96.

12. Rapparini C. Occupational HIV infection among health care workers exposed to blood and body fluids in Brazil. Am J Infect Control 2006; 34: 237-40.

13. Secretaria de Estado da Saúde do Rio Grande do Sul. Seção de Controle das Doenças Sexualmente Transmissíveis e da Aids. Boletim Epidemiológico AIDS. Disponível em: URL: http:/ /www.saude.rs.gov.br/aids/boletim_aids.php. Acesso em 12 de fevereiro de 2011.

14. Silveira TR, Fonseca JC, Rivera L, Fay OH, Tapia R, Santos JI, Urdeneta E, Clemens SAC. Hepatitis B seroprevalence in Latin America. Pan Am J Public Health 1999; 6: 378-83.

15. Niu MT, Colemam P, Alter MJ. Multicenter study of hepatitis $C$ virus infection in chronic hemodialysis patients and hemodialysis center staff members. Am J. Kideney Dis 1993; 22: 568-73.
16. São Paulo (Estado). Secretaria de Estado da Saúde. Programa Estadual de DST/AIDS. Divisão de Vigilância Epidemiológica. Boletim Epidemiológico SINABIO. Dos acidentes com material biológico: prevenir é preciso. Boletim epidemiológico C. R. T. DST/AIDS C. V. E. jan. 2004; 2:16.

17. São Paulo (Estado). Secretaria de Estado de Saúde de São Paulo. Programa Estadual de DST/AIDS. Divisão de Vigilância Epidemiológica. SINABIO: vigilância de acidentes com material biológico. Boletim Epidemiológico C. R. T. - DST/AIDS C. V. E. 2002; 1: 39-19:20

18. Rapparini C, Vitória MAA, Lara LTR. Recomendações para atendimento e acompanhamento de exposição ocupacional a material biológico: HIV e Hepatites B e C. Brasília, [s.d] [cited 2010 abr 16]. Available from: http: http:// www.crorj.org.br/biosseguranca/ manual_ acidentes.pdf.

19. Lopes LKO, Tipple AFV, Damasco SN et al. Atendimento aos profissionais vítimas de acidente com material biológico em um hospital de doenças infecto-contagiosas. Rev. Eletrônica Enferm, Goiânia 2004; 6: 324-9.

20. Marziale MHP. Subnotificação de acidentes de trabalho perfurocortante entre trabalhadores de enfermagem. Rev. Bras. Enferm. Brasília 2003; 56: 164-8.

21. Canini SRMS, Gir E, Hayashida M, Machado AA. Acidentes perfurocortantes entre trabalhadores de enfermagem de um hospital universitário do interior paulista. Rev Lat Am Enfermagem, Ribeirão Preto 2002; 10:172-8.

22. Brevidelli MM, Cinciarullo TI. Aplicação do modelo de crenças em saúde na prevenção dos acidentes com agulha. Rev. Saúde Pública, São Paulo 2001; 35:193-201.

23. Braga D. Acidente de trabalho com material biológico em trabalhadores da equipe de enfermagem do Centro de Pesquisas Hospital Evandro Castro. [Dissertação de Mestrado], Rio de Janeiro: Fundação Oswaldo Cruz, Escola Nacional de Saúde Pública; 2000

24. Napoleão AA, Robazzi MLCC, Marziale MHP et al. Causas de subnotificação de acidente de trabalho entre trabalhadores de enfermagem. Rev Lat Am Enfermagem, Ribeirão Preto 2000; 8: 119-20.

25. Caixeta RB, Barbosa-Branco A. Acidente de trabalho, com material biológico, em profissionais de saúde de hospitais públicos do Distrito Federal, Brasil, 2002/2003. Cad. Saúde Pública, Rio de Janeiro 2005; 21: 737-46.

26. Marziale MHP, Nishimura KYN, Ferreira MM. Riscos de contaminação ocasionados por acidentes de trabalho com material perfurocortante em trabalhadores de enfermagem. Rev Lat Am Enfermagem, Ribeirão Preto 2004; 12: 36-42.

27. Sêcco IAO, Leroux AMR, Santos CF et al. Epidemiologia dos acidentes de trabalho com material biológico na equipe de enfermagem de hospital público do Paraná. UNOPAR Cient Ciênc Biol Saúde, Londrina 2002; 4: 37-43.

28. Abreu ES. Avaliação histórica e do seguimento dos profissionais da saúde com exposição aos materiais biológicos atendidos no Instituto Emilio Ribas no período de 1985 a 2001 [Dissertação de Mestrado], São Paulo: Programa de PósGraduação em Ciências, Coordenação de Controle de Doenças, Secretaria de Estado da Saúde de São Paulo; 2005.

29. Sarquis LMM, Felli VEA, Miranda FMD et al. A adesão ao protocolo de monitoramento dos trabalhadores de saúde após exposição a fluidos biológicos: uma problemática vivenciada em um ambulatório de saúde do trabalhador no Paraná. Cogitare Enferm. Curitiba 2005; 10: 47-53. 
30. Nishide VM, Benatti MCC, Alexandre NMC. Ocorrência de acidente de trabalho em uma unidade de terapia intensiva. Rev Lat Am Enfermagem, Ribeirão Preto 2004; 12: 204-11.

31. Benatti MCC. Acidentes de trabalho entre trabalhadores de enfermagem de um hospital universitário. Rev Esc Enferm. USP, São Paulo 2001; 35:155-62.

32. Bellissimo-Rodrigues WT, Bellissimo-Rodrigues F, Machado AA. Occupational exposure to biological fluids among a cohort of Brazilian dentists. Int Dent J. Geneva 2006; 56: 332-7.

33. Canini SRMS, Gir E, Machado AA. Accidents with potentially hazardous biologycal material among workers in hospital supporting services. Rev Lat Am Enfermagem, Ribeirão Preto 2005;13:496-500.

34. Rodrigues NML. Conhecimento e adesão às medidas de precauções padrão dos funcionários do serviço de higiene e limpeza hospitalar. [Dissertação de Mestrado], Escola Paulista de Medicina, Universidade Federal de São Paulo, São Paulo; 2001.

35. Orestes-Cardoso SM, Farias ABL, Pereira MRMG et al. Acidentes perfurocortantes: prevalência e medidas profiláticas em alunos de odontologia. Rev. Bras. Saúde Ocup. São Paulo 2009; 34: 6-14.
36. Brasil. Ministério da Saúde. Secretaria de Vigilância em Saúde. Programa Nacional de DST e Aids. Recomendações para Terapia Anti-Retroviral em Adultos e Adolescentes Infectados pelo HIV. Brasília: Ministério da Saúde, 2006. [cited 2010 abr 18]. Available from: http://www.news.med.br/p/ +quot+recomendacoes+para+terapia+an-1301.html.

37. Gir E, Costa FPP, Silva AM. Enfermagem frente a acidentes de trabalho com material potencialmente contaminado na era do HIV. Rev. Esc. Enferm. USP, São Paulo 1998; 32: 267-72.

38. Marziale MHP, Rodrigues CM. A produção científica sobre os acidentes com material perfurocortante entre trabalhadores de enfermagem. Rev Lat Am Enfermagem, Ribeirão Preto 2002 ; $10: 571-7$.

39. Osório C, Machado JMH, Gomez CM. Proposição de um método de análise coletiva dos acidentes de trabalho no hospital. Cad. Saúde Pública, Rio de Janeiro 2005; 21: 517-24.

40. Figueiredo GM, Oliveira M. (Coord.). Guia de orientações técnicas: Hepatites B e C. São Paulo; 2002.

41. Gerberding JL. Management of occupational exposures to blood-borne viruses. N Engl J Med, United States 1995; 332: 444-51. 